\title{
Multiconfiguration time-dependent Hartree approach for electron-nuclear correlation in strong laser fields
}

\author{
Chirag Jhala* and Manfred Lein \\ Institut für Theoretische Physik and Centre for Quantum Engineering and Space-Time Research (QUEST), Leibniz, Universität Hannover, \\ Appelstraße 2, D-30167 Hannover, Germany \\ (Received 2 April 2010; published 24 June 2010)
}

\begin{abstract}
The multiconfiguration time-dependent Hartree approach is applied to study the electron-nuclear correlation in the dynamics of molecules subject to strong external laser fields, using the example of a model hydrogen molecular ion. The ground state of the system is well described by as few as two single-particle functions per degree of freedom. A significantly larger but moderate number of configurations is required to predict laser-induced fragmentation probabilities and high-order harmonic generation spectra accurately, showing that the correlation between electronic and nuclear degree of freedom is strongly increased by the presence of the laser field.
\end{abstract}

DOI: 10.1103/PhysRevA.81.063421

PACS number(s): $33.80 . \mathrm{Rv}, 42.65 . \mathrm{Ky}, 33.20 . \mathrm{Xx}$

\section{INTRODUCTION}

The field strength of a high-intensity laser pulse can be comparable to or larger than typical atomic or molecular binding forces [1,2]. This leads to a multitude of effects involving high excitation, ionization, and fragmentation. Among these phenomena, high-order harmonic generation (HHG) [3] has emerged as a particularly important field of research. HHG spectra from molecules provide information about the molecular structure [4] and have been used to construct electron orbitals [5-7]. HHG has been qualitatively explained by the three-step model [8] as follows. First, the electron tunnels out of the atomic or molecular system as the laser field suppresses the potential barrier. The electron is subsequently accelerated by the field and driven back toward the parent ion. In the final step the electron recombines with the parent ion by emitting the kinetic energy accumulated during its excursion together with the binding energy into a photon.

For an accurate description of molecules in a strong field, both the response of the electrons and also the nuclear degrees of freedom must be taken into account. Ideally, the dynamics of the different degrees of freedom are treated fully quantum mechanically. A classical description of the nuclei or using the Born-Oppenheimer (BO) approximation cannot fully explain the complex interplay between the different degrees of freedom in situations where electrons are excited into high-lying bound or continuum states. The standard method of solving the full time-dependent Schrödinger equation uses a representation of the wave function and Hamiltonian in an appropriate product basis. This approach works very well for small systems but the required computational resources grow exponentially with increasing number of degrees of freedom. Even simple systems such as the $\mathrm{H}_{2}{ }^{+}$molecular ion pose a challenge to theoretical description when they are subject to intense laser pulses because large grids or basis sets are required to capture the ionization and fragmentation dynamics in the nonperturbative regime of laser-molecule interactions. For $\mathrm{H}_{2}{ }^{+}$, full non-BO quantum mechanical simulations incorporating the nuclear motion have been reported only for

*Chirag.Jhala@itp.uni-hannover.de the case of frozen rotational degree of freedom. For the usual infrared fields, such simulations are limited to the case of the molecular axis aligned parallel to the laser field [9-11] while the case of arbitrary oriented three-dimensional $\mathrm{H}_{2}{ }^{+}$ has been treated in the fixed-nuclei approximation [12,13]. Recently, the combined treatment of arbitrary orientation and nuclear motion has been reported [14,15] for driving fields in the high-frequency (extreme ultraviolet) regime, which are computationally less demanding than the low-frequency fields. Another set of recent calculations [16,17] includes the full rovibrational quantum mechanical motion of the nuclei, but uses a BO expansion including the three lowest electronic states.

As an alternative to the direct solution of the Schrödinger equation, approximate methods have been developed that maintain a fully quantum mechanical picture while alleviating the problem of exponentially growing computational requirements. One such method is the time-dependent Hartree (TDH) approach [18], in which the wave function is approximated as one Hartree product. The required effort to solve the system is reduced significantly but at the cost of losing the correlation between the degrees of freedom completely. The TDH approach does not perform well in describing correlated multielectron dynamics and does not offer a way to converge to the exact result, which makes it hard to determine the quality of a calculation. To overcome these problems, the multiconfiguration time-dependent Hartree (MCTDH) approach [19-21] has been proposed. The MCTDH wave function is a sum of Hartree products. Here, each Hartree product can be understood as a configuration and the sum covers all possible configurations that arise from the set of single-particle functions (which are time dependent themselves). MCTDH has been applied extensively to study the nuclear dynamics of multidimensional systems. Systems of identical particles can be studied with the help of the MCTDH method but this involves a large amount of redundant coefficients in the multiconfigurational expansion [22]. A variant that is applicable to the case of many-electron systems is known as the multiconfiguration time-dependent Hartree-Fock (MCTDHF) method [23]. MCTDHF differs from the MCTDH in the explicit antisymmetrization of the electronic wave function. A TDH approach for identical particles neglects exchange 
effects while this is not the case in MCTDH and MCTDHF. Note that for distinguishable degrees of freedom (the normal case in MCTDH applications), there is no exchange effect. The term configuration in the MCTDH context should be used with care when comparing with the configuration-interaction (CI) method of atomic structure theory. In the CI method, the wave function is written as a linear combination of configuration state functions built from a given set of spin orbitals. Different configuration state functions describe the excitation of single or multiple electrons from occupied orbitals to the unoccupied orbitals. Time-dependent CI calculations have been carried out previously [24,25]. It is important to note that in this method the expansion coefficients are time dependent while the configurations remain fixed. This is in contrast to MCTDH where the configurations are allowed to evolve in time as well. Moreover, the CI wave function does not necessarily include all possible configurations, but may be restricted to certain types of excitation (single, double, etc.). In the present work, we apply the multiconfiguration theory for the first time to the case of coupled electronic and nuclear motion. We use a onedimensional (1D) model $\mathrm{H}_{2}{ }^{+}$system [26-32] to compare exact simulations to the MCTDH method with various numbers of configurations. For laser parameters that are typically used in present-day experiments, we find that a moderate number of single-particle functions is sufficient to provide accurate quantitative results for fragmentation probabilities and $\mathrm{HHG}$ spectra. Atomic units are used throughout this article.

\section{MULTICONFIGURATION TIME-DEPENDENT HARTREE APPROACH}

The MCTDH approach [20] for an $f$-dimensional system approximates the wave function $\Psi$ by a sum of Hartree products. The multiconfigurational wave function ansatz reads

$$
\Psi\left(Q_{1}, \ldots, Q_{f}, t\right)=\sum_{j_{1}=1}^{n_{1}} \cdots \sum_{j_{f}=1}^{n_{f}} A_{j_{1} \cdots j_{f}}(t) \prod_{\kappa=1}^{f} \varphi_{j_{\kappa}}^{(\kappa)}\left(Q_{\kappa}, t\right)
$$

where $Q_{1}, \ldots, Q_{f}$ are the particle coordinates, the $A_{j_{1} \ldots j_{f}}$ denote the MCTDH expansion coefficients, and $\varphi_{j_{\kappa}}^{(\kappa)}$ are the $n_{\kappa}$ single-particle functions (SPFs) for each degree of freedom $\kappa$. We simplify the notation by introducing a composite index $J=\left(j_{1} \ldots j_{f}\right)$ and the configurations $\Phi_{J}$ :

$$
A_{J}=A_{j_{1} \cdots j_{f}}, \quad \Phi_{J}=\prod_{\kappa=1}^{f} \varphi_{j_{\kappa}}^{(\kappa)} .
$$

The numbers $n_{\kappa}$ of SPFs that are used to build the configurations can be chosen differently for each degree of freedom. However, each of these numbers $n_{\kappa}$ should obey the condition,

$$
n_{\kappa}^{2} \leqslant \prod_{\kappa^{\prime}=1}^{f} n_{\kappa^{\prime}}
$$

Otherwise, there will be redundant configurations. The condition $n_{1}=n_{2}$ follows for two degrees of freedom. For the present calculation, we thus have $f=2$ and $n_{1}=n_{2}=n$, and the respective number of configurations is $n^{2}$.

Using the multiconfigurational ansatz in the Dirac-Frenkel variational principle [33],

$$
\left\langle\delta \Psi\left|H-i \partial_{t}\right| \Psi\right\rangle=0,
$$

one obtains, under suitable constraints [20], a set of coupled equations of motion for the expansion coefficients and for the set of SPFs:

$$
\begin{gathered}
i \dot{A}_{J}=\sum_{L}\left\langle\Phi_{J}|H| \Phi_{L}\right\rangle A_{L}, \\
i \dot{\boldsymbol{\varphi}}^{(\kappa)}=\left(1-P^{(\kappa)}\right)\left(\rho^{(\kappa)}\right)^{-1}\langle\boldsymbol{H}\rangle^{(\kappa)} \boldsymbol{\varphi}^{(\kappa)} .
\end{gathered}
$$

Here a vector notation has been adopted for the SPFs with $\varphi^{(\kappa)}=\left(\varphi_{1}^{(\kappa)}, \ldots, \varphi_{n_{\kappa}}^{(\kappa)}\right)^{T}$. Furthermore, $P^{(\kappa)}=$ $\sum_{j=1}^{n_{\kappa}}\left|\varphi_{j}^{(\kappa)}\right\rangle\left\langle\varphi_{j}^{(\kappa)}\right|$ is the projector on the space spanned by the SPFs for the $\kappa^{\text {th }}$ degree of freedom, $\rho^{(\kappa)}$ is the density matrix, and $\langle\boldsymbol{H}\rangle^{(\kappa)}$ is the matrix of mean fields. For a detailed derivation and discussion of Eqs. (5) and (6), see [20]. The propagation of the wave function is expected to become more accurate as the number of $n_{\kappa}$ is increased. Thus, for $n_{\kappa} \rightarrow \infty$, the MCTDH wave function is expected to converge toward the exact one. Setting $n_{1}=\cdots=n_{f}=1$ one arrives at the time-dependent Hartree method, which approximates the total wave function by one product of SPFs. For large systems, the required effort to solve the MCTDH equations of motion is typically much less than in the direct solution of the time-dependent Schrödinger equation.

TABLE I. Ground-state energy for the 1-D $\mathrm{H}_{2}{ }^{+}$molecular ion obtained from the Hartree, MCTDH, and exact calculations. All numbers are in atomic units.

\begin{tabular}{lllcrr}
\hline \hline & \multicolumn{3}{l}{ Hartree } & \multicolumn{3}{c}{ MCTDH } & Exact \\
\cline { 2 - 6 } & \multicolumn{4}{c}{ Number of single-particle functions } \\
\cline { 2 - 6 } & $n_{\kappa}=1$ & 2 & 4 & 8 & $\infty$ \\
\hline$-E_{0}$ & 0.77484 & 0.77636 & 0.77638 & 0.77638 & 0.77638 \\
$\langle\widehat{T}\rangle$ & 0.08439 & 0.08393 & 0.08392 & 0.08392 & 0.08392 \\
$-\left\langle W_{\mathrm{en}}\right\rangle$ & 1.24113 & 1.24098 & 1.24095 & 1.24095 & 1.24094 \\
$\left\langle W_{\mathrm{nn}}\right\rangle$ & 0.38190 & 0.38069 & 0.38065 & 0.38065 & 0.38065 \\
$\langle R\rangle$ & 2.6293 & 2.6431 & 2.6435 & 2.6435 & 2.6435 \\
\hline \hline
\end{tabular}



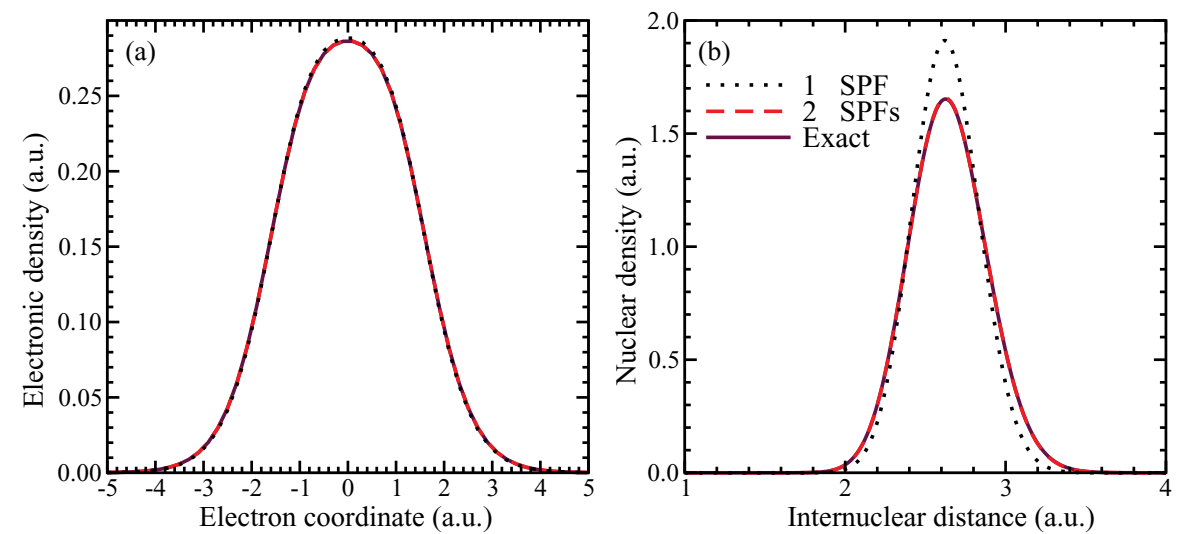

FIG. 1. (Color online) The groundstate (a) electronic density and (b) nuclear density obtained for the $1 \mathrm{D} \mathrm{H}_{2}{ }^{+}$molecular ion using Hartree, MCTDH, and exact calculations.

\section{THE MODEL SYSTEM}

We consider a one-dimensional $\mathrm{H}_{2}{ }^{+}$molecular ion since it is the simplest possible system with coupled electronic and nuclear motion. The model system is characterized by the Hamiltonian,

$$
\widehat{H}=\widehat{T}_{n}+\widehat{T}_{e}+W_{\mathrm{en}}(x, R)+W_{\mathrm{nn}}(R)+q_{e} x E(t),
$$

with $\widehat{T}_{n}=-\frac{1}{2 \mu_{n}} \frac{\partial^{2}}{\partial R^{2}}$ and $\widehat{T}_{e}=-\frac{1}{2 \mu_{e}} \frac{\partial^{2}}{\partial x^{2}}$. Here, $x$ and $R$ denote the electron coordinate and the internuclear distance. $\mu_{n}$ and $\mu_{e}$ are the reduced masses of nuclei and electron, respectively, and $q_{e}=\frac{2 m_{n}+2}{2 m_{n}+1}$ is the reduced charge. Instead of bare Coulomb potentials, the soft-core potentials,

$$
\begin{gathered}
W_{\mathrm{en}}(x, R)=-\frac{1}{\sqrt{\left(x-\frac{R}{2}\right)^{2}+a_{e}}}-\frac{1}{\sqrt{\left(x+\frac{R}{2}\right)^{2}+a_{e}}}, \\
W_{\mathrm{nn}}(R)=\frac{1}{\sqrt{R^{2}+a_{n}}},
\end{gathered}
$$

are used with $a_{e}=1$ and $a_{n}=0.03$. The laser field is given by

$$
E(t)=E_{0} f(t) \sin (\omega t),
$$

where $\omega$ is the laser frequency and $E_{0}$ is the peak amplitude. In all our calculations, we use a laser wavelength of $800 \mathrm{~nm}$. The envelope function, $f(t)=\sin ^{2}\left(\frac{\pi}{2} \frac{t}{T_{\text {ramp }}}\right)$, is used to ramp up the laser field from zero to the maximal field strength at time $t=T_{\text {ramp. }}$. For the time-dependent calculations, the wave function $\Psi(x, R, t)$ is represented on a grid with $N_{R} \times N_{x}=256 \times 2048$ points. The spatial step sizes for the internuclear distance and for the electron coordinate are $d R=0.1$ a.u. and $d x=0.2$ a.u., respectively. For the calculation of ground-state properties the step sizes were $d R=0.05$ a.u. and $d x=0.1$ a.u. A part of our calculations was carried out using the Heidelberg MCTDH package [34].

\section{RESULTS}

\section{A. Ground-state calculation}

In this section we compare the ground-state properties obtained from the Hartree, MCTDH, and exact calculations. The ground state is obtained via imaginary time propagation [35]. Table I shows the total ground-state energy $E_{0}$ obtained from the different methods. The kinetic energy $\langle\widehat{T}\rangle$, electron-nuclear interaction energy $\left\langle W_{\text {en }}\right\rangle$, and nuclear-nuclear interaction energy $\left\langle W_{\mathrm{nn}}\right\rangle$ are the components that contribute to $E_{0}$. The Hartree approach underestimates the absolute value of the ground-state energy while the MCTDH approach gradually improves and converges toward the exact results.

In Figs. 1(a) and 1(b), we plot the ground-state electronic density $\rho_{e}$ and nuclear density $\rho_{n}$ defined as

$$
\begin{aligned}
& \rho_{e}(x)=\int_{0}^{R_{\max }}|\Psi(x, R)|^{2} d R, \\
& \rho_{n}(R)=\int_{-x_{\max }}^{x_{\max }}|\Psi(x, R)|^{2} d x,
\end{aligned}
$$

where $R_{\max }=25.6$ a.u. and $x_{\max }=204.8$ a.u. are the end of the grid in nuclear and electronic direction, respectively. The disagreement between the Hartree and exact calculations is clearly visible in the nuclear density plot. The deviation of Hartree electronic density from the exact calculation is very minute. The MCTDH approach leads to almost exact
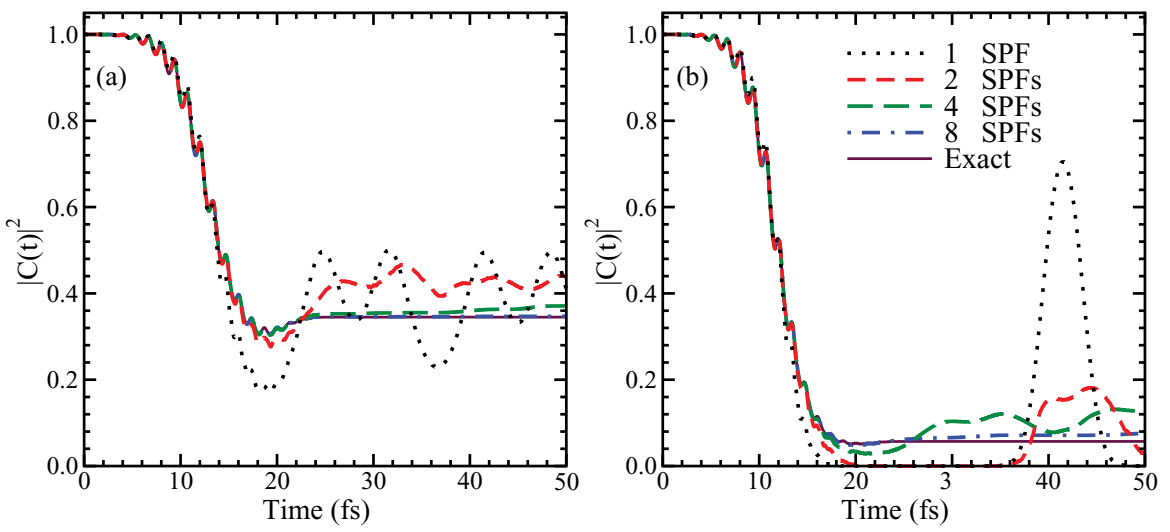

$063421-3$
FIG. 2. (Color online) Modulus squared $|C(t)|^{2}$ of the autocorrelation function plotted for intensities (a) $2 \times 10^{14}$ $\mathrm{W} / \mathrm{cm}^{2}$ and (b) $3 \times 10^{14} \mathrm{~W} / \mathrm{cm}^{2}$ as a function of time obtained for $1 \mathrm{D} \mathrm{H}_{2}{ }^{+}$ using the Hartree, MCTDH, and exact calculations. 

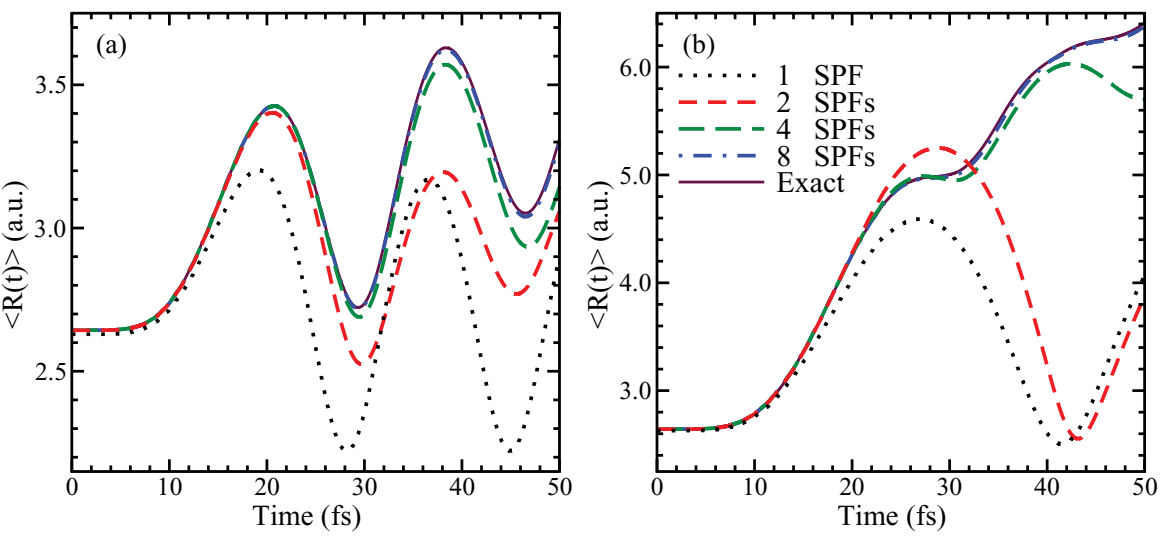

FIG. 3. (Color online) Time evolution of the mean internuclear distance $\langle R\rangle(\mathrm{t})$ for the intensities (a) $2 \times 10^{14} \mathrm{~W} / \mathrm{cm}^{2}$ and (b) $3 \times 10^{14} \mathrm{~W} / \mathrm{cm}^{2}$ obtained for $1 \mathrm{D}$ $\mathrm{H}_{2}{ }^{+}$using Hartree, MCTDH, and exact calculations. results. Only on a closer inspection of Fig. 1(b), we find that the two-SPF nuclear density deviates slightly from the exact calculation. For more than two SPFs, the MCTDH densities are indistinguishable from the exact densities on the scale of the figure.

\section{B. Dynamics in the presence of a laser field}

The model system is subjected to laser pulses with intensities $2 \times 10^{14} \mathrm{~W} / \mathrm{cm}^{2}$ and $3 \times 10^{14} \mathrm{~W} / \mathrm{cm}^{2}$. A function with five optical cycles turn-on and turn-off times is used as the field envelope. The pulse length is $26.7 \mathrm{fs}$ ( 10 cycles) and the total propagation time is $50 \mathrm{fs}$ ( 19 cycles). To avoid reflections from the boundary, complex absorbing potentials [36] have been applied.

The autocorrelation function $C(t)=\langle\Psi(0) \mid \Psi(t)\rangle$ is defined as the overlap of $\Psi(t)$ with the initial ground state $\Psi(0)$. In Figs. 2(a) and 2(b), we plot $|C(t)|^{2}$ for the two intensities as a function of time. This quantity can be viewed as the probability that the system is still in the ground state at time $t$. The Hartree result closely follows the exact calculation initially. Around the end of the pulse and at later times, the Hartree result deviates strongly from the exact calculation. In fact, the probability fails to remain constant during the field-free evolution after the pulse. The two-SPF case shows improvement compared to the Hartree calculation but differs strongly from the exact calculation. With increasing numbers of SPFs, the exact result is approached.

Figure 3 shows the time-dependent expectation value $\langle R\rangle=\int_{0}^{R_{\max }} \rho_{n}(R) R d R$ of the internuclear distance. For the intensity $2 \times 10^{14} \mathrm{~W} / \mathrm{cm}^{2}$ [Fig. 3(a)], the laser field causes the molecule to stretch at the beginning of the pulse. As the field strength is moderate, the internuclear distance starts oscillating rather than showing dissociation. Compared to the exact results, the Hartree ansatz reproduces the main features of the dynamics qualitatively. It follows an initially increasing and oscillatory behavior of $\langle R\rangle(t)$. The amplitude of the oscillation is underestimated while its frequency is overestimated. The MCTDH approach successfully reproduces the features of exact calculation. For the intensity $3 \times$ $10^{14} \mathrm{~W} / \mathrm{cm}^{2}$ [Fig. 3(b)], the exact and the MCTDH calculations show initial stretching of the molecule and fragmentation. It is interesting to see that the two-SPF case qualitatively reproduces the exact calculation for the weaker field but fails for the stronger field. The Hartree calculation shows an oscillatory behavior with higher amplitude than in the lower-intensity case. The oscillatory result indicates that the stability of the system against fragmentation is overestimated by the Hartree ansatz. This is consistent with the too-narrow shape of the nuclear ground-state density [Fig. 1(b)].

In Fig. 4, the time evolution of the nuclear density is plotted for the laser intensity $3 \times 10^{14} \mathrm{~W} / \mathrm{cm}^{2}$. The eightSPF case [Fig. 4(c)] and the exact calculation [Fig. 4(d)] exhibit dissociation. Here the nuclear density splits into two parts: one representing the part of the wave function which remains bound in some superposition state, while the other one corresponds to a dissociative packet. The two-SPF case [Fig. 4(b)] shows almost negligible dissociation, similar to the Hartree method [Fig. 4(a)]. The time evolution of electronic density is plotted in Fig. 5. The slowly outgoing electronic wave packets observed in the center of the plot [Figs. 5(c) and 5(d)] are associated with a dissociation process: as the nuclei move apart, the electron follows the one or the other. The separation of dissociating wave packets from the ground-state region is not visible in the Hartree method [Fig. 5(a)] and two-SPF case [Fig. 5(b)].
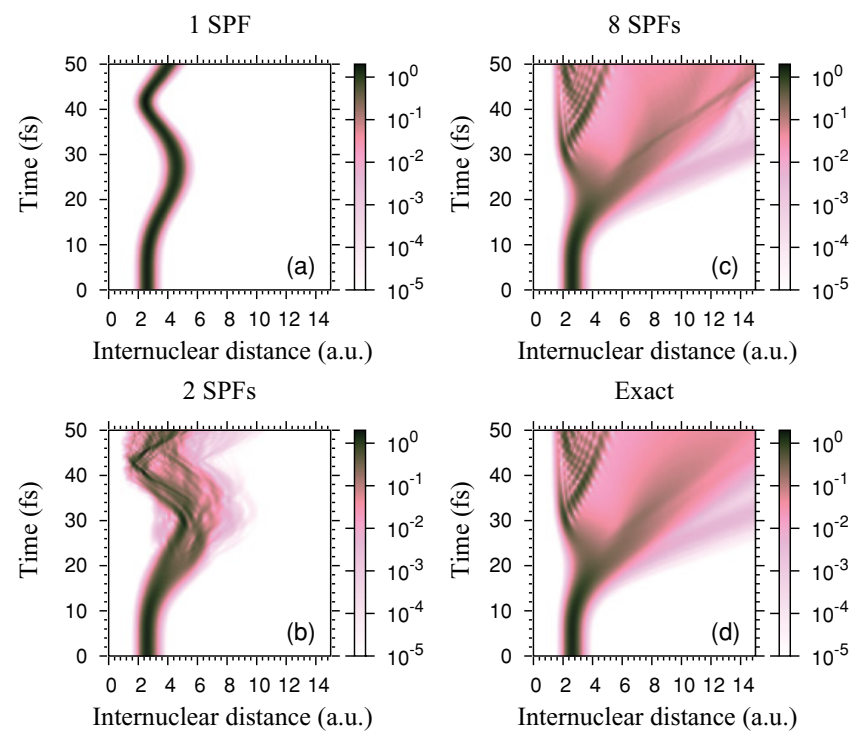

FIG. 4. (Color online) Time evolution of the nuclear density obtained for $1 \mathrm{D} \mathrm{H}_{2}{ }^{+}$in a laser pulse with intensity $I=3 \times$ $10^{14} \mathrm{~W} / \mathrm{cm}^{2}$. 

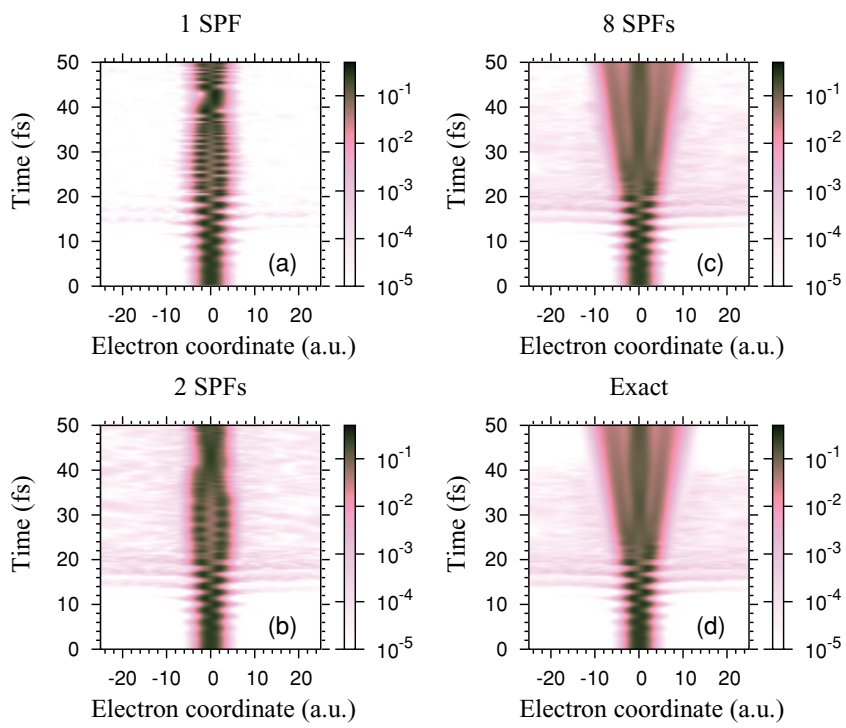

FIG. 5. (Color online) Time evolution of the electronic density obtained for $1 \mathrm{D}_{2}{ }^{+}$in a laser pulse with intensity $I=3 \times 10^{14}$ $\mathrm{W} / \mathrm{cm}^{2}$.

The calculation of fragmentation probabilities is of particular interest in the simulations of strong-field phenomena. We can distinguish two types of fragmentation: (i) dissociation into $\mathrm{H}+\mathrm{H}^{+}$, denominated in the following as photodissociation [37], and (ii) fragmentation into $\mathrm{H}^{+}+\mathrm{H}^{+}$, known as Coulomb explosion [37,38]. In principle, the calculation of fragmentation probabilities requires projection of the manybody wave function on all the continuum states. Alternatively, one can use a geometrical picture to obtain approximate results. It rests on the simplified assumption that all bound states of the system under consideration are contained inside some finite volume of the numerical grid. In our calculation, we set $R_{\text {box }}=8$ a.u. and $x_{\text {box }}=25$ a.u. to define the probabilities of survival (SU), photodissociation (PD), and Coulomb explosion (CE) as follows:

$$
P_{\mathrm{SU}}=\int_{0}^{R_{\mathrm{box}}} d R \int_{-x_{\mathrm{box}}}^{x_{\mathrm{box}}} d x|\Psi(x, R, t)|^{2}
$$

$$
\begin{gathered}
P_{\mathrm{PD}}=\int_{R_{\mathrm{box}}}^{R_{\max }} d R \int_{-x_{\mathrm{box}}}^{x_{\mathrm{box}}} d x|\Psi(x, R, t)|^{2}, \\
P_{\mathrm{CE}}=1-P_{\mathrm{SU}}-P_{\mathrm{PD}} .
\end{gathered}
$$

In Fig. 6 we plot $P_{\mathrm{SU}}, P_{\mathrm{CE}}$, and $P_{\mathrm{PD}}$ as functions of time for the intensities $2 \times 10^{14} \mathrm{~W} / \mathrm{cm}^{2}$ and $3 \times 10^{14} \mathrm{~W} / \mathrm{cm}^{2}$. When the model system is subjected to the field intensity $2 \times 10^{14} \mathrm{~W} / \mathrm{cm}^{2}$, the system remains in some vibrationally excited bound state most of the time. The fragmentation of the molecule is very small. The exact calculation shows that roughly $94 \%$ of the molecule withstands fragmentation, while the Hartree result exhibits very little fragmentation [Fig. 6(a)]. The same is true for the two-SPF case. In Fig. 6(b), we see that the one-SPF case shows a slight decrease in $P_{\mathrm{CE}}$ at long times. This is purely an artifact of one-SPF calculation and can be attributed to the "shrinking" of the wave function after the end of the pulse. The photodissociation probability is practically zero for the one-SPF case and is not visible in Fig. 6(c). The two-SPF case shows a minute improvement. With increasing numbers of SPFs, the deviation becomes less and the MCTDH results converge toward the exact results. For the intensity $3 \times 10^{14} \mathrm{~W} / \mathrm{cm}^{2}$ [Figs. 6(d)-6(f)], the MCTDH calculations reproduce the results of the exact calculation well. As before, the one-SPF approach fails to give a proper account of electron-nuclear correlation in the presence of an external field [39].

The harmonic spectrum is obtained by taking the modulus squared of the Fourier transform of the time-dependent dipole acceleration $a(t)$ [40]:

$$
\begin{gathered}
\mu_{e} a(t)=\left\langle\Psi(t)\left|\nabla W_{\mathrm{en}}+q_{e} E(t)\right| \Psi(t)\right\rangle, \\
\widetilde{a}(\omega)=\frac{1}{\sqrt{2 \pi}} \int a(t) e^{i \omega t} d t .
\end{gathered}
$$

For the calculation of HHG we have used 10-cycle pulses with two-cycle (5.3 fs) $\mathrm{sin}^{2}$-shaped ramping-on and ramping-off functions. HHG spectra obtained for the model system are plotted in Fig. 7. We find well-defined peaks at odd harmonics order (i.e., harmonic frequencies that are odd multiples of the incident laser frequency). The peaks in the HHG spectra from the MCTDH approach with four SPFs and eight SPFs
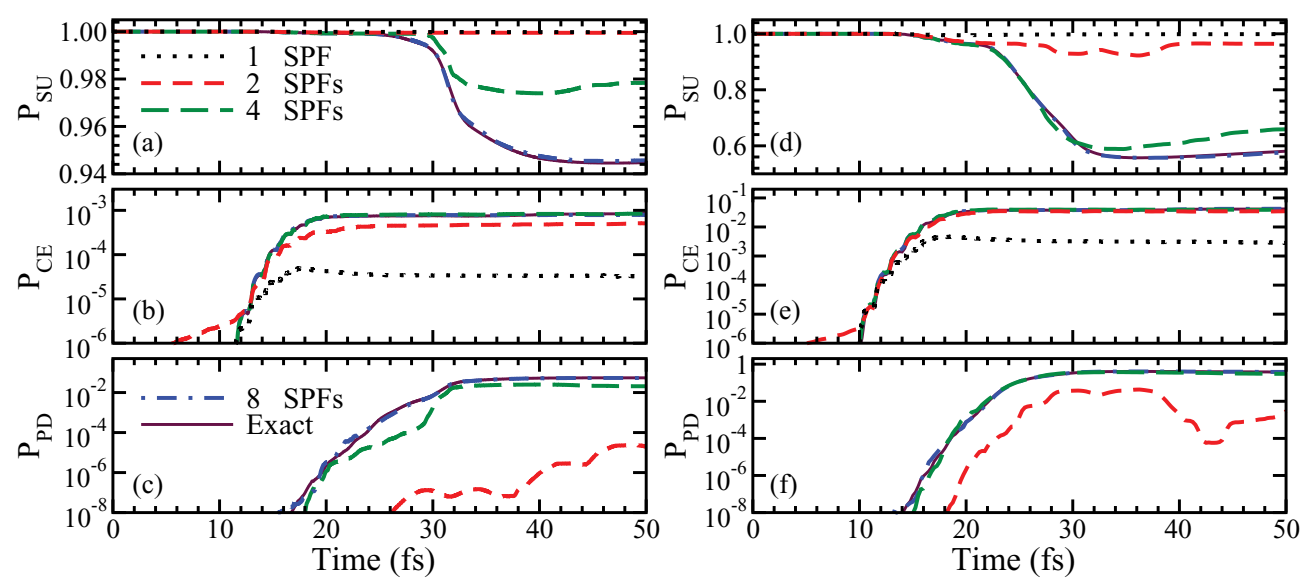

FIG. 6. (Color online) Survival (SU), Coulomb explosion (CE), and photodissociation (PD) probabilities obtained for $1 \mathrm{D} \mathrm{H}_{2}{ }^{+}$in fields of intensities $2 \times 10^{14} \mathrm{~W} / \mathrm{cm}^{2}$ [panels (a)-(c)] and $3 \times 10^{14} \mathrm{~W} / \mathrm{cm}^{2}$ [panels (d)-(f)] as functions of time. 


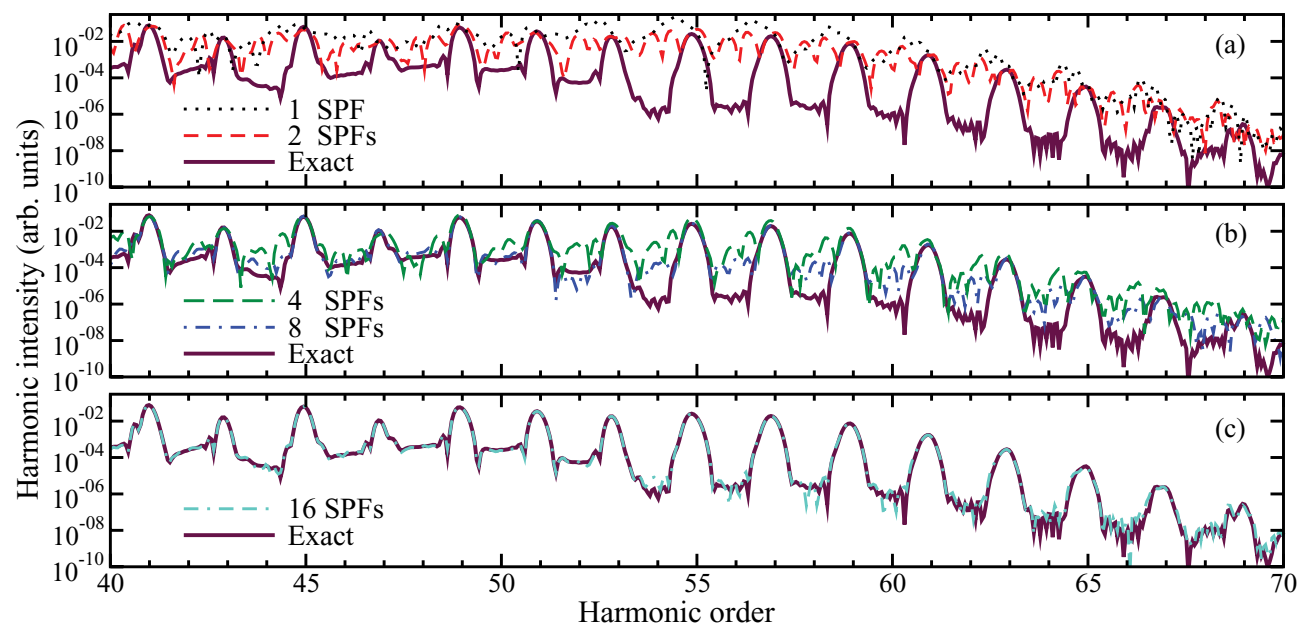

FIG. 7. (Color online) HHG spectra obtained for $1 \mathrm{D}_{2}{ }^{+}$in a laser pulse with intensity $I=3 \times 10^{14} \mathrm{~W} / \mathrm{cm}^{2}$.

[Fig. 7(b)] lie at the same position as in the exact spectra. The Hartree calculation produces peaks, but it is difficult to distinguish them in the plateau region [see Fig. 7(a)]. Apparently, a sufficiently large number of SPFs is needed to produce the shape of the HHG peaks accurately, and the 16-SPF result is very close to the exact result [see Fig. 7(c)].

\section{CONCLUSION}

We have studied the electron-nuclear correlation in the presence of strong laser fields using the MCTDH formalism using the example of the one-dimensional $\mathrm{H}_{2}{ }^{+}$molecular ion. Although rather simple, the model system shows a rich dynamics and exhibits many of the salient features that are characteristic of the behavior of diatomic molecules in intense fields (ionization, photodissociation, Coulomb explosion). We have demonstrated that the Hartree approach fails while the performance of the MCTDH approach converges toward the exact results with increasing numbers of single-particle functions. It allows one to systematically improve the accuracy toward the exact result. Besides, we have shown that the number of configurations required for numerically converged results increases for increasing peak intensity of the infrared laser pulse (i.e., the correlation between electronic and nuclear motion is increased by the field). Nevertheless, the number of required SPFs is moderate. While two to four SPFs are sufficient to calculate ground-state properties accurately, eight to $16 \mathrm{SPFs}$ yield good results for the laser-driven system. We conclude that the MCTDH method is a practicable approach for the treatment of coupled electronic and nuclear motion.

\section{ACKNOWLEDGMENTS}

C.J. is grateful to Hans-Dieter Meyer for introducing him to the MCTDH method. We thank the Deutsche Forschungsgemeinschaft for funding the Centre for Quantum Engineering and Space-Time Research (QUEST).
[1] D. Batani, C. J. Joachain, S. Martellucci, and A. N. Chester, Atoms, Solids, and Plasmas in Super-Intense Laser Fields (Kluwer Academic/Plenum, New York, 2001).

[2] F. Grossmann, Theoretical Femtosecond Physics (Springer, Berlin Heidelberg, 2008).

[3] F. Krausz and M. Ivanov, Rev. Mod. Phys. 81, 163 (2009).

[4] M. Lein, J. Phys. B 40, R135 (2007).

[5] J. Itatani, J. Levesque, D. Zeidler, H. Niikura, H. Pépin, J. C. Kieffer, P. B. Corkum, and D. M. Villeneuve, Nature 432, 867 (2004).

[6] E. V. van der Zwan, C. C. Chirilă, and M. Lein, Phys. Rev. A 78, 033410 (2008).

[7] S. Haessler et al., Nature Phys. 6, 200 (2010).

[8] P. B. Corkum, Phys. Rev. Lett. 71, 1994 (1993).

[9] V. Roudnev, B. D. Esry, and I. Ben Itzhak, Phys. Rev. Lett. 93, 163601 (2004).

[10] S. Chelkowski, T. Zuo, O. Atabek, and A. D. Bandrauk, Phys. Rev. A 52, 2977 (1995).
[11] F. He, C. Ruiz, and A. Becker, Phys. Rev. Lett. 99, 083002 (2007).

[12] G. L. Kamta and A. D. Bandrauk, Phys. Rev. A 71, 053407 (2005).

[13] T. K. Kjeldsen, L. B. Madsen, and J. P. Hansen, Phys. Rev. A 74, 035402 (2006).

[14] M. Førre and H. Bachau, Phys. Rev. A 77, 053415 (2008).

[15] S. X. Hu, L. A. Collins, and B. I. Schneider, Phys. Rev. A 80, 023426 (2009).

[16] M. Winter, R. Schmidt, and U. Thumm, Phys. Rev. A 80, 031401(R) (2009).

[17] M. Winter, R. Schmidt, and U. Thumm, New J. Phys. 12, 023020 (2010).

[18] A. D. McLachlan, Mol. Phys. 8, 39 (1964).

[19] H.-D. Meyer, U. Manthe, and L. S. Cederbaum, Chem. Phys. Lett. 165, 73 (1990).

[20] M. H. Beck, A. Jäckle, G. A. Worth, and H.-D. Meyer, Phys. Rep. 324, 1 (2000). 
[21] H.-D. Meyer, F. Gatti, and G. A. Worth, Multidimensional Quantum Dynamics: MCTDH Theory and Applications (WileyVCH, Weinheim, 2009).

[22] O. E. Alon, A. I. Streltsov, and L. S. Cederbaum, J. Chem. Phys. 127, 154103 (2007).

[23] J. Zanghellini, M. Kitzler, T. Brabec, and A. Scrinzi, J. Phys. B 37, 763 (2004).

[24] T. Klamroth, Phys. Rev. B 68, 245421 (2003).

[25] N. Rohringer, A. Gordon, and R. Santra, Phys. Rev. A 74, 043420 (2006).

[26] K. C. Kulander, F. H. Mies, and K. J. Schafer, Phys. Rev. A 53, 2562 (1996).

[27] W. Qu, Z. Chen, Z. Xu, and C. H. Keitel, Phys. Rev. A 65, 013402 (2001).

[28] S. Chelkowski, C. Foisy, and A. D. Bandrauk, Phys. Rev. A 57, 1176 (1998).

[29] G. L. Ver Steeg, K. Bartschat, and I. Bray, J. Phys. B 36, 3325 (2003).

[30] B. Feuerstein and U. Thumm, Phys. Rev. A 67, 043405 (2003).

[31] C. Liu, Z. Zeng, P. Wei, P. Liu, R. Li, and Z. Xu, Phys. Rev. A 81, 033426 (2010).
[32] M. Lein, T. Kreibich, E. K. U. Gross, and V. Engel, Phys. Rev. A 65, 033403 (2002).

[33] J. Broeckhove, L. Lathouwers, E. Kesteloot, and P. Van Leuven, Chem. Phys. Lett. 149, 547 (1988).

[34] G. A. Worth, M. H. Beck, A. Jäckle, and H.-D. Meyer, computer code, The MCTDH Package, Version 8.2, University of Heidelberg, Heidelberg, Germany, 2000; H.-D. Meyer, computer code, The MCTDH Package, Version 8.3 (2002), Version 8.4 (2007). [http://mctdh.uni-hd.de].

[35] R. Kosloff and H. Tal-Ezer, Chem. Phys. Lett. 127, 223 (1986).

[36] R. Kosloff and D. Kosloff, J. Comp. Phys. 63, 363 (1986).

[37] D. Pavičić, Coulomb Explosion and Intense-Field Photodissociation of Ion-Beam $\mathrm{H}_{2}{ }^{+}$and $\mathrm{D}_{2}{ }^{+}$, Ph.D. thesis, LudwigMaximilians-Universität München, 2004.

[38] H. Stapelfeldt, E. Constant, and P. B. Corkum, Phys. Rev. Lett. 74, 3780 (1995).

[39] T. Kreibich, R. van Leeuwen, and E. K. U. Gross, Chem. Phys. 304, 183 (2004).

[40] K. Burnett, V. C. Reed, J. Cooper, and P. L. Knight, Phys. Rev. A 45, 3347 (1992). 